FUNDING AND DISCLOSURE

The authors declare no conflict of interest.

Bishop GA, Berbari NF, Lewis J, Mykytyn K (2007) Type III adenylyl cyclase localizes to primary cilia throughout the adult mouse brain. J Comp Neurol 505: 562-571.

Brailov I, Bancila M, Brisorgueil MJ, Miquel MC, Hamon M, Verge D (2000). Localization of 5- $\mathrm{HT}(6)$ receptors at the plasma membrane of neuronal cilia in the rat brain. Brain Res $\mathbf{8 7 2}$ 271-275.

Green JA, Gu C, Mykytyn K (2012). Heteromerization of ciliary $\mathrm{G}$ protein-coupled receptors in the mouse brain. PLOS ONE 7: e46304.

Green JA, Mykytyn K (2010). Neuronal ciliary signaling in homeostasis and disease. Cell Mol Life Sci 67 3287-3297.

Handel M, Schulz S, Stanarius A, Schreff $M$, Erdtmann-Vourliotis M, Schmidt $\mathrm{H}$ et al (1999). Selective targeting of somatostatin receptor 3 to neuronal cilia. Neuroscience 89: 909-926.

Lee JE, Gleeson JG (2011). Cilia in the nervous system: linking cilia function and neurodevelopmental disorders. Curr Opin Neurol 24: 98-105.

Neuropsychopharmacology Reviews (2014) 39, 244-245; doi: I 0. I 038/npp.20I3.203

\section{When Synaptic Proteins Meet the Genome: Transcriptional Regulation in Cell Death and Plasticity by the Synapto-Nuclear Messenger Jacob}

Neurons express more genes than any other cell type and it is therefore unlikely that synapto-nuclear $\mathrm{Ca}^{2+}$ signaling alone can explain the specific genomic response to the plethora of extracellular stimuli that control gene expression. Possible candidates for encoding of signals at the origin and later decoding at a nuclear destination are synapto-nuclear protein messenger. These proteins translocate to the nucleus in a stimulus-dependent manner, where they can regulate via their nuclear target interactions very specific aspects of gene expression (Jordan and Kreutz, 2009). However, this type of signaling is challenged by space and time constraints. Synapses are localized far away from the nucleus and synaptic signals are very labile and rapidly degraded. On top the minute quantities of signaling molecules that can be released from synaptic sites are greatly diluted in relation to the entire volume of the dendritic arborization or nucleus. A precise machinery is therefore required to deliver the signal to nuclear target sites, but unfortunately very little is known on just how protein messenger can translocate several hundred micrometers, avoid degradation, retain signal and do this in a reliable manner.

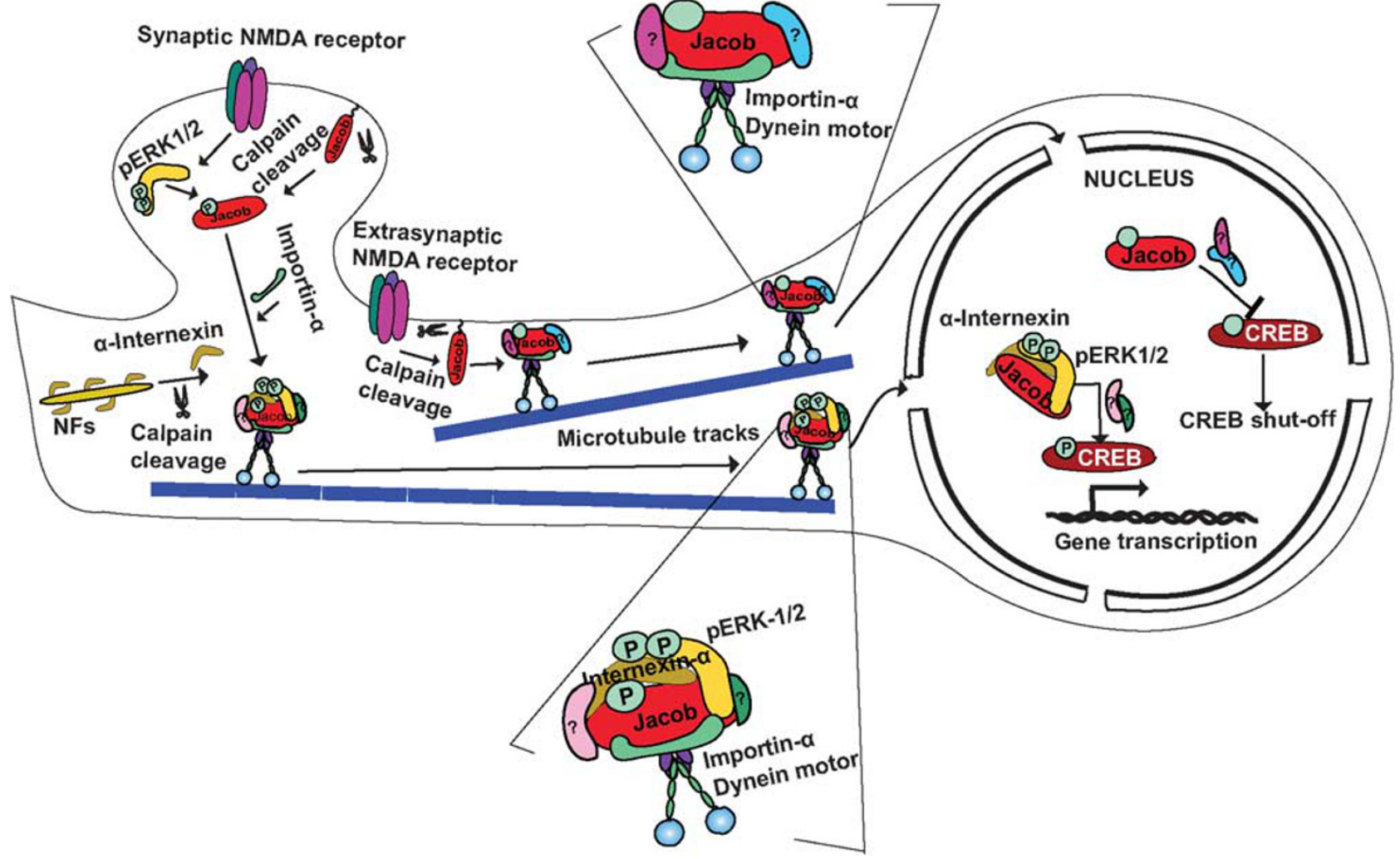

Figure 1. Schematic illustrating the encoding of the origin of synaptic and extrasynaptic NMDAR signals by Jacob. Neuronal activity leads to Calpainmediated cleavage of $\alpha$-internexin and the myristoylated $\mathrm{N}$ terminus of Jacob. High but not low frequency stimulation of synaptic NMDAR results in ERK activation, ERK binding, and phosphorylation of the serine180 in Jacob. This is then followed by recruitment of C-terminal fragments of $\alpha$-internexin or a tighter assembly of an already preformed complex, which is then efficiently protected against phosphatase activity analogous to a mechanism demonstrated by Perlson et al (2005) for the retrograde transport of pERK after axon injury. In the nucleus, this complex can dock to CREB and potentially other sites and will enhance plasticity and cell survival promoting gene expression. Following the extrasynaptic NMDAR activation, ERK is not activated and remains outside of the nucleus, while non-phosphorylated Jacob will dock yet unknown protein components to CREB that eventually induce CREB shut-off and cell death. 
$N$-methyl-D-aspartate receptors (NMDAR) make an essential contribution to activity-dependent gene expression in learning and memory. However, NMDAR are present at both synaptic and extrasynaptic sites, and the subcellular localization of each receptor profoundly and differentially affects the nuclear response to its activation. Activation of synaptic NMDAR induces the expression of cell survival and plasticity genes, while their extrasynaptic counterparts primarily drive the expression of cell death genes, linking the pathway to disease (Hardingham and Bading, 2010). An unresolved issue is how can the distant nucleus discriminate between synaptic and extrasynaptic NMDAR-induced signals?

Jacob is a synapto-nuclear messenger, and previous work has shown that extrasynaptic NMDAR activation induces nuclear translocation of Jacob, which results in sustained dephosphorylation and transcriptional inactivation of the transcription factor CREB, a loss of synaptic contacts, a retraction of dendrites and eventually cell death (Dieterich et al, 2008). However, Jacob also transits to the nucleus of CA1 neurons, following induction of Schaffer collateral-dependent long-term potentiation (LTP) but not long-term depression (LTD), and hence acts as a messenger for both synaptic and extrasynaptic NMDAR pathways (Behnisch et al, 2011). How does the protein get to the nucleus and what is the molecular basis for these different functions after nuclear import of Jacob? Neuronal importins are present in axons, dendrites, and synapses and they can associate with a dynein motor for active retrograde transport along microtubuli to the nucleus. Jacob utilizes this transport system after activation of both type of receptors and in a recent study we found that Jacob, following its nuclear import, can encode the synaptic and extrasynaptic origin of NMDAR signals (Karpova et al, 2013). ERK1/2kinase binding and ERK-dependent phosphorylation of the serine180 residue in Jacob encodes synaptic but not extrasynaptic NMDAR activation.
A stable trimeric complex with proteolytically cleaved fragments of the neurofilament $\alpha$-internexin is formed, which protects Jacob and active ERK against phosphatase activity during retrograde transport. In the nucleus, this signalosome-like complex enhances 'plasticity-related' and 'CREBdependent' gene expression as well as synaptic strength. It appears that Jacob operates as a mobile hub that docks NMDA receptor-derived signalosomes to nuclear target sites and it will be interesting to clarify the molecular identity of these complexes, because they are attractive targets for pharmacological interventions in activity-dependent gene transcription.

\section{ACKNOWLEDGEMENTS}

This study was supported by the DFG (SFB 779/TPB8), DIP grant, CBBS Magdeburg, MC-ITN NPlast, and the Leibniz Foundation (Pakt für Forschung).

\section{Sujoy Bera ${ }^{1}$ and Michael R Kreutz ${ }^{1}$ \\ ${ }^{1}$ RG Neuroplasticity, Leibniz-Institute for \\ Neurobiology, Magdeburg, Germany \\ E-mail: kreutz@lin-magdeburg.de}

\section{FUNDING AND DISCLOSURE}

The authors declare no conflict of interest.

Behnisch T, Yuanxiang P, Bethge P, Parvez S, Chen Y, Yu J et al (2011). Nuclear translocation of Jacob in hippocampal neurons after stimuli inducing longterm potentiation but not long-term depression. PLoS One 6: e17276.

Dieterich DC, Karpova A, Mikhaylova M, Zdobnova I, König I, Landwehr $\mathrm{M}$ et al (2008). Caldendrin-Jacob: a protein liaison that couples NMDA receptor signalling to the nucleus. PLOS Biol 6: e34.

Hardingham GE, Bading H (2010). Synaptic versus extrasynaptic NMDA receptor signalling: implications for neurodegenerative disorders. Nat Rev Neurosci 11: 682-696.

Jordan BA, Kreutz MR (2009). Nucleocytoplasmic protein shuttling: The direct route in synapse-to-nucleus signaling. Trends Neurosci 32: 392-401.

Karpova A, Mikhaylova M, Bera S, Bär J, Reddy PP, Behnisch Tet al (2013). Encoding and transducing the synaptic or extrasynaptic origin of NMDA receptor signals to the nucleus. Cell 152: 1119-1133.

Perlson E, Hanz S, Ben-Yaakov K, Segal-Ruder Y, Seger R, Fainzilber M (2005). Vimentin-dependent spatial translocation of an activated MAP kinase in injured nerve. Neuron 45: 715-726.

Neuropsychopharmacology Reviews (2014) 39, 245-246; doi: 10.1 038/npp.2013.204

\section{Maintaining and Modifying Connections: Roles for Axon Guidance Cues in the Mature Nervous System}

Upon reaching its target, an axon differentiates to form synaptic connections. Remarkably, recent studies have revealed that proteins initially identified as axon guidance cues are re-emerging as regulators of synaptic plasticity in the adult brain. Canonical axon guidance proteins such as semaphorins, ephrins, and slits, and the prototypical myelin-associated inhibitors of axon regeneration, MAG, Nogo, and OMgp, have been found to influence circuit remodeling and synaptic plasticity in the mature nervous system (Mironova and Giger, 2013). Following a study by Horn et al (2013), the netrin receptor DCC now joins this list.

Mechanisms that direct neural development have long been suggested to regulate plasticity in the mature brain. Cajal's (1911) proposal that chemotropism might direct axon extension in the embryo included a hypothesized attractant for commissural axons secreted by floor plate cells in the embryonic spinal cord. In a stunning act of prescience, Cajal (1911) suggested that his putative chemoattractant might also influence synaptic plasticity and learning and memory: 'The ability of neurons to grow in an adult and their power to create new associations can explain learning.' $\mathrm{He}$ speculated that 'the mechanisms are probably chemotactile like the ones we observed during histogenesis of the spinal cord'.

Netrins were the first identified floor plate-derived axonal chemoattractants (Lai Wing Sun et al, 2011). Although shown to regulate synaptogenesis during development in Caenorhabditis elegans and Drosophila melanogaster, a role for netrins at synapses in the mature vertebrate CNS had not been investigated. Horn et al 